\title{
CLOUD COMPUTING LOADING TIME OVER DIFFERENT OPERATING SYSTEMS
}

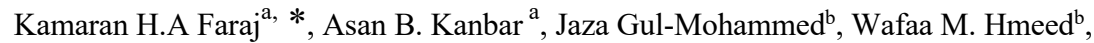 \\ Shagul F. Karim ${ }^{\mathrm{c}}$ \\ ${ }^{a}$ Dept. of Computer Science, Cihan University-Sulaimaniya, University of Sulemani, Iraq, (kamaranfaraj@yahoo.com) \\ ${ }^{a}$ Dept. of Computer Science, Cihan University-Sulaimaniya, Iraq, (asan.baker@sulicihan.edu.krd) \\ ${ }^{b}$ Faculty of Engineering, University of Sulemani, Sulemani, Iraq, (jeza.gul@univsul.edu.iq) \\ ${ }^{b}$ Dept. of Computer Science, Cihan University-Sulaimaniya, Iraq, (wafaa.mustafa@sulicihan.edu.krd) \\ ${ }^{\mathrm{c}}$ College of Agricultural engineering science, University of Sulemani, Sulemani, Iraq, (shagul.karim@univsul.edu.iq)
}

Received: Sep., 2020 / Accepted: Nov., 2020 / Published: Dec.,2020

https://doi.org/10.25271/sjuoz.2020.8.4.756

\begin{abstract}
:
Since the traditional time loading (TTL) very primitive before the era of information communication technology (ICT) and it was really not depended on the result of time-loading due to the old version of computer architecture (i.e. serial processing). Nevertheless, the parallel processing systems open a wide area of researching for electronic time loading (ETL) over different operating systems by programing languages (i.e. python or private home page (Php)). The electronic time loading (ETL) for cloud Computing (CC) is a hot experimental topic. ETL for CC is not only one parameter (i.e. a web technologies type or a web applications type or an infrastructures type or an architectures type). Moderately, the term CC refers to the evolution of the information technology (IT). As we realized the ETL is very important for reducing time wasting. The reducing time-waste loading over different web operating systems or CC is a target in this paper. Finally, this paper test the Electronic Loading Time of CC over different operating systems with different types of network (i.e. public and private) discovering the least ETL. Hence the benchmarking TTL is not applicable (N/A) due to the activity from a person to others is very changeable and not depended on it at all. This paper shows the total time and load time over different OS in seconds, and find out the least time loading required this work is a good solution of the response time over different operating system in open source-LOS and non-open source WOS.
\end{abstract}

KEYWORDS: Cloud Computing, Electronic Time Loading, Traditional Time Loading, Parallel Processing Systems, Operating Systems.

\section{INTRODUCTION}

Nowadays, the importance of measuring performance as HPC use cases broaden, more supercomputers are being built to faster and more powerful specifications (Scot Schultz October 19, 2020).

Most of results of time loading are relay on ETL not TTL and the benchmark of proposed WebOS or CloudOS is very important factor to enhance the performance. In generally, both Linux operating system and windows operating system benchmarking is over several types of CloudOS that our proposed system investigate only with two popular types of WebOs especially EyeOs and Lucid desktop. Thus, both of the mentioned WebOS types are tested on two methods which are PrWebOS and PuWebOS. Both of eyeOS and lucid ( Chunnjie LUO, Jianfeng ZHAN, Zhen JIA, Lei WANG, Gang LU, Lixin ZHANG, Chng-Zhong XU, Ninghui 2012). The designed eyeOS and lucid are separately run; first over the Linux OS-puWebOS and second time over windows OS-prWebOS. It is very essential to be mention that the system was planned to facilitate the implementation of the needs of users at the lowest cost, fastest time and without users need to carry PCs all time as this system enables the pass process at anytime and anywhere. This is a benchmark that improved and makes life easier for users. The two parameter namely Benchmark of CC system in higher ranking results for $\mathrm{CC}$ systems and data processing application (Chunjie LUO, Jianfeng ZHAN, Zhen JIA, Lei WANG, Gang LU, Lixin ZHANG, Cheng-Zhong XU, Ninghui SUN,2012) is related to our proposed WebOS or CloudOS, Over the past decade technologies have made great progresses due to the extremely significant and revolutionary ICT facilities is undoubtedly the Internet. The rapid development of networked and mobile computing, as demonstrated with the ever growing Internet (or Web) has led to a global infrastructure, as well as to the introduction of innovative information communication technology (ICT) functionality $(\mathrm{H}$. Unger, P.Kroph,2000). A Web OS can be defined as user interface (UI) that authorize the individuals to access applications keep fully or partly on the net (Shubham kumar sahu, 2Dr. R.K. Khare,2019). The facility of the Internet appears to be a very alike of only extremely large distributed architecture that must be visited or opened to a widely used by a Web Operating System (H.Unger, P.Kroph,2000). The traditional method of OS had been modernized by the emergence of ICT for example the Internet. In the past few years, the Internet has noticeably enhanced the face of TOS and the ways organizations about the OS functionality. In the coming years, WebOS are to continue their explosive growth in real life of computer science as a relation of Web technology and operating system. Modern Load Sharing and Load Balancing systems can enhance this relation when more of These systems have a least cost-performance relation and can avoid high costs for the installation of parallel supercomputers (H. Unger, P.Kroph,2000). The WebOS functions are independent platform (or crosswise platforms) - from any device with Internet connected. Since cloud computing works only with internet, this leads to the development of WebOS. Cloud computing creates co-operation in an easier way and reduce platform-incompatibility difficulties (D.Kirshna, R.Meshach,2013). The WebOSs are run on the browser platform-independent, since browsers are constructed to function across different TOS. In a single machine client the

* Corresponding author

This is an open access under a CC BY-NC-SA 4.0 license (https://creativecommons.org/licenses/by-nc-sa/4.0/) 
TOS types for example (windows, Linux, Unix and Mac) must be selected only one by the user exactly before loading OSs in a single machine, thus it's a dependent. But WebOs easily support every OS types without selection and it's independent. Platform as a Service (PaaS) is one of three fundamental service ideal of CC that our proposed system investigate to enhance the TOS functionality and etc. PaaS in our proposed system is distribution of middleware directed towards developers with a platform that combines the entire development cycle, including hosting, testing, and deployment of web applications (i.e. eyeOS, luiced) (J.Duan, P.Fasker, A.Fesak, T.Stuart,2012). Cloud Computing is an internet based network technology that shared a fast growth in communication technology by providing service to customers of various requirements with the aid of online computing resources. It has supplies of both hardware and software applications along with software development platforms and testing tools as resources (Mishra SK, Sahoo B, Parida PP ,2018), Since Server operating system (SOS) and Web operating system (Web0S) (A.Vahdat, E.Belani, P.Eastham, C.Yoshikawa, T.Anderson, D.Culler, M.Dahlin,1998) are construction to allows a new model for/by the Internet services as a middleware on any sorts of networked interconnects client and server based.

The networked communication category's examples are private network (PriN) - Wireless LAN and Public network (PubN) (K.Faraj, T. Ahmad, H.Shareef, A.Najib, G,Najeeb,2015). Our planned system is mainly concentrated on the Private Web0S and Public Web0S. The procedure of software application with not installation in any personal computer (PC) is marvel for the reason that the CC help it very possible in digitalized today's world. It stores both primary and secondary in the any computer because information data is on centralized datacenter and sited outside your own place or PC. In TOSs, TOSs users must to install OS applications software to computer separated to any other computer, either server or clients. The TOS is separation installed OS, but Web0s or CC is integrated only one OS and shred by others clients. The only required web browser, internet facility and installed OS over servers. Thus, the applications would exist on computer's hard disk drive. A Web OS might look a lot like a TOS, but it doesn't manage user computer's hardware or software. A Web OS permits user to access applications stored not on user computer, but on the Web. The applications exist completely or in part on Web servers within a specific provider network. When user saves information in an application, user might not store it on a personal computer. Instead, user saves the information to databases connected to the Internet. Some Web operating systems also give user the option to save information to user local hard disk drive (R.Mondal, D.Sarddar,2016), and which is in a high level of security. The smart of Web0S is Instead of being distributed or fixed to a single location for example PC the services can dynamically organize responsibilities onto Internet computing resources-client. The term of Web0S is a software platform that interacts with the user over a web browser and does not depend on any particular (R. Sharma,2013) TOS. Both TOS and Web Operating System (WOS) are not as same as or replacement of each other's, but there are two different functionality and operation (R.Sachdeva, P.Sharma, N.Kataria,2015). However TOS and WOS are supplementing each other. It's difficult for any computer instrument to operate without OSs. The OSs is a part of program software that systematizes and it's a medium to controls both hardware and software. The Web0S communicate straight with computer hardware and serve as a platform for different applications. Computer depends on its OS to function, such as; Windows, Linux, UNIX or Mac OSX. However, A Web0S is a user interface (UI) that allows individuals to Access applications that stored only or in part on the Web [SSK15]. The OS of WOS that installed in server side is a main, although the TOS that connect to WOS via internet is a secondary OS. The implementation of WOS is manufactured on the Internet facility and distributed computing. The objective of this Web0S is to distribute the complete benefit of the World Wide Web (WWW). The WebOS embrace mechanisms for managing client or data management in data center, remote procedure execution, persistent storage, confirmation and secure (A.Tripathi,2014). Scopes of the principles are satisfied feature of superb quality $(Q)$, enterprise $\mathrm{Q}$, user $\mathrm{Q}$, and Organization Q. With wide-ranging indicators of these scopes together and their check list the web originators and developers can build a best of $\mathrm{Q}$ websites to develop the Eservice (L.Hasan, E. Abuelrub, 2011). For instance one type the Web0S is Lucid Desktop (formerly known as the Psych Desktop) is built on a base on private home page (PHP) version5 and is a presume-oriented web desktop service, it can be installed on to a web server like Eye0S, and is easy to use (R. Sharma,2013). However, the eyeOS is an open source web desktop following the CC idea it is mainly written in PHP, hypertext mark-up language (HTML), and cascading style sheet (CSS). It drives as a platform for web applications written using the eyeOS Toolkit. It includes a Desktop environment with applications and system services. It is reachable by movable devices via its mobile frontend. The examples of movable devices for our proposed system frontend are smart phone, iPad and phone-mobile. The innovative contribution is to analysis for incredible performance between categories namely (PrWebOS) - Wireless LAN and Public network (PuWebOS). The capability of smart phone PrWebOS-wireless is much better than the smart phone PuWebOS-Wireless. Generally both public and private WebOS are parts of CC. The services provided by a public cloud are offered over the Internet and are preserved and operated by a cloud provider. Some examples include services aimed at the general public, such as online photo storage services, e-mail services, or social networking sites and services for enterprises can also be offered in a public cloud. In a private cloud, the cloud infrastructure is operated solely for a specific organization, and is managed by the organization or a third party (D. Therrien,2016). The cloud has three forms of models: Software as a Service (SaaS), Platform as a Service (PaaS), and Infrastructure as a Service (IaaS). In SaaS, the user uses the collections of application running in the cloud. While in PaaS the user use the tools provided by the platform from classes libraries and other language supports. However, the user has control over the operating system and the application deployed in the IaaS with changing the Infrastructure components and configurations $(\mathrm{H}$. Abusaimeh,2014). WebOS is mostly focused on enabling different kinds of phone calls, messaging services, settings and device information, multitasking, and synchronizing phone book data and other virtual resources (H.Steen, Tiger,2014). Finally, the WebOS is one of cloud computing implementation (A.Tripathi,2014). and also its one of the most recent and popular computer science topic by ICT. WebOS is offered for cloud computing and it is potential evaluated when compared with TOS and WebOS types for example; EyeOS and Luicd over private and public WebOS.

\section{RELATED WORKS}

A comparative analysis of Windows, Unix, Linux, Mac, Android and iOS operating systems performed (A. Adekotujo ,A. Odumabo ,A. Adedokun ,O. Aiyeniko,2020) based on the OS features and their strengths and weaknesses In our proposed system we also made a comparison and focus on public and private WebOS and measured the loading time over different operating systems.

The study (Joel Scheuner, Philipp Leitner, 2019), discourses tools for performance benchmarking IaaS clouds and provoke 
the importance of cloud benchmarking, show the execution of a simple IaaS benchmark in a public cloud provider using the CWB web interface, and develop a more advanced benchmark with configuration management integration To support service selection, researchers and practitioners conduct cloud performance benchmarking by measuring and objectively comparing the performance of different providers and configurations (e.g., instance types in different data center regions) the limitation of this work is not taking the private cloud in consideration that's in our proposed system prwebOS and puWebOs are utilized in order to provide a better service.

The research in (Rajat, Ajay Kumar Bharti, 2018) focuses to determine the best service broker policy and scheduling algorithm considering the distance of data center. Considering the frequency of factors appearing from the review work it has been inferred that the cloud response time is very crucial for the cloud performance and selected for as a related work for our study.

the work in (Shubham kumar sahu, Dr. R.K. Khare,2019) they performed a survey to collect the knowledge and make comparisons between the available web based operating system / webtop ,briefly mentioning the points and features of different webOs and also the history of web os , their types, working methods and how useful. This study have not considered the chrome OS because it needs to be installed in the system, it is a cloud system but that need to be installed inside the system and besides comparing all of the above the EyeOs and the Oneye is best the provide a great features, they are open source, you can install them on your web hosting with php version 5.6, just the main drawback is they have stopped developing now, in future we need a complete system that can be accessed via web browser and offers all the features and functionality that a user needs, yes chrome os has that all but you need to install that in a system, eyes has almost features but with various bugs and biggest thing to look upon is the security as they are all online always that means hackers will definitely try to hack and because it's in cloud it less secure compared to traditional operating systems available, this work gives information about webOs which was beneficial in our work. The study conducted by (R.Mondal, D.Sarddar,2016) realized the Server operating system (SOS) and Web operating system (WebOS) are construction to allows a new model for/by the Internet services as a middleware on any sorts of networked interconnects client and server based. Web OS has the potential of being an important distributed computing system for the Internet. It promises supporting applications that are geographically distributed with high reliability, security, scalability, and manageability. Some research and prototyping of Web OS have been underway to overcome some of the challenges and difficulties pertaining to the design and implementation of such a system. This study does not mention the parameter of execution time which is very important, In our work we worked on open source and non- open source operating system taking the execution time in confederation.

\section{ARCHITECTURE OF THE PROPOSED SYSTEM}

In general, the proposed system was designed to run either on prWebOS or puWebOS. Both methods are client/server based computers network. However, the TOS in a single computer is not a C/S and N/A. PrWebOS and puWebOS architecture are on $\mathrm{C} / \mathrm{S}$ base networked computers with any network scales. Web0S or cloudOS; There are several areas of concern when first setting up prWebOS and puWebOS systems: 1. Back-end (IaaS) 2. Web application Data (SaaS$\mathrm{PaaS})$ 3. Front-end (SaaS). The back-end is a backbone of any new technology systems especially for WebOS. The developers should be sure that the established back-end is secure and is working faultlessly. The design of back-end module has highest priority in the system establishment schedule, most of the errors in the systems caused by the inaccuracy of back-end module. MySQL is an outstanding tool to implement the database system. The technology used in the proposed system to link the database to front-end interface is PHP with Windows OS as a server using of XAMP application for the prWebOS and Linux- Ubuntu 14.04 OS as a server using of LAMP application for puWebOS. Figure1. shows the prWebOS and puWebOs over different cloud OS. The Notpad++ has been used as an editor for typing codes of the proposed system. Almost all of the work of cloud WebOS takes place on the server, except the specific application called "webserver", which is responsible for communication with the browser. A relational database server stores whatever information the application requires. One of the easy languages could be used to handle requests between the web server and the database server is PHP by apache webserver.

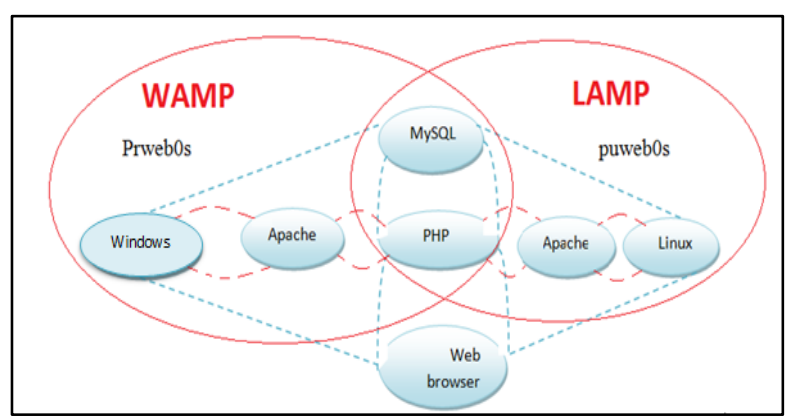

Figure 1. Shows the prwebOS and puWebOs over different OS

\subsection{Software Interfaces:}

There are three different methods of Web0S that related to our proposed system which are:

A) Private PrwebOS: the facility is located on virtually separate infrastructure which is designed by us without of need the Internet facility because it is only intranet with specific limitation. PrwebOS is need the hardware and software requirements. Where the user device is connect to router and can easily access to the web application server which distributes the signal to each Customer of income to the Web application, that are link by relying on IP address, this method also called (intranet or offline network ) with several advantages of this method:-

1) Select (limited) number of users that allow only those who are inside the building within the network, to communicate.

2) The increase of the efficiency, performance, quality and speed communication as the communication is much better

3) The distance and numbers of users' limitation in PrwebOS help us to be more confidential and security of information of users in the backend-database system. Figure (2) shows the PrwebOS.

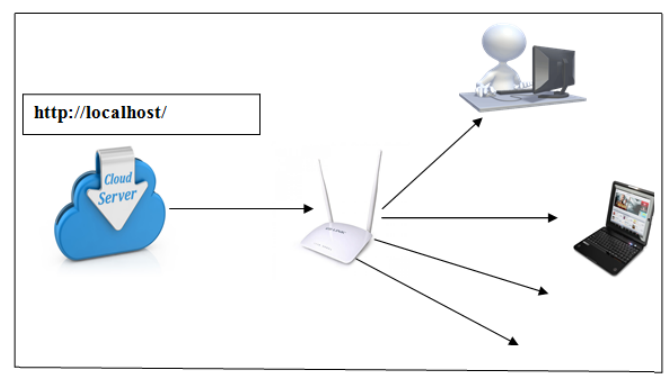

Figure 2.Shows the private WebOS 
B) Public PuWebOs: The accessibility of puWebOs by anyone via the Internet facility with the shared infrastructure as a multiple consumers. Hence, it's a strong reason of the public cloud attitudes of higher security risk. Generally when the numbers of users are increase the level of security is decrees. Figure (3) shows the PuWebOs.

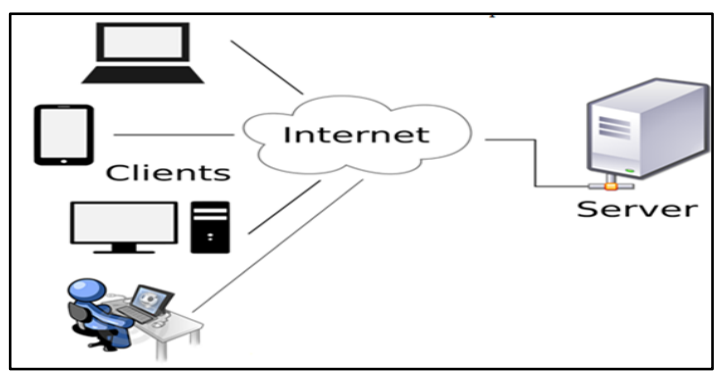

Figure 3.Shows the public Web0S

Figure (4) shows the direct relation between number of users to security for PrwebOS and PuWebOs. The only condition for puWebOs is availability of internet facility if compared to the prwebOS.

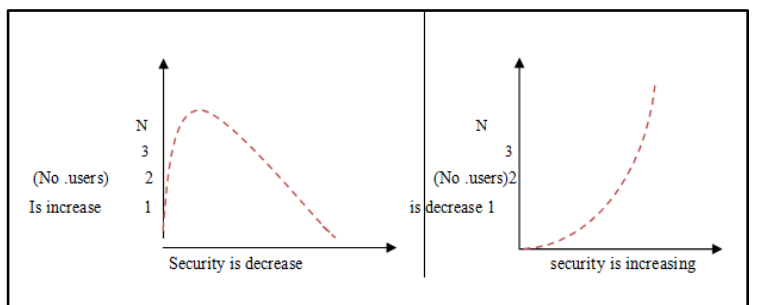

Figure 4. Direct relation between numbers of users to security forPrwebOS and PuWebOs

Due to the puWebOs drawback when compared to prwebOS for example: -

1. Because the number of users more Therefore, we believe that the confidentiality and protection Information and data system is weak because of the intrusion that can occur from any third person or from any user. Its main aim follow-up information from other users of web system .

2. Also, the distance to be non-limited and may be a large cross Continents Because it depends on the presence of the Internet in any place was that allow user to login and access to application.

C) ChWebOS: in our proposed system prwebOS and puWebOs are utilized in order to provide a better service. Blended of prwebOS and puWebOs create chweb0S. The interior users of application as a staffs traffic police is an example of prwebOS, but the exterior consumer that use the traffic police application by internet facility is an example of puWebOs. Our proposed system is chweb0S because of the use of both web0S types. Figure(5) shows Blended of prwebOS and puWebOs create chweb0S

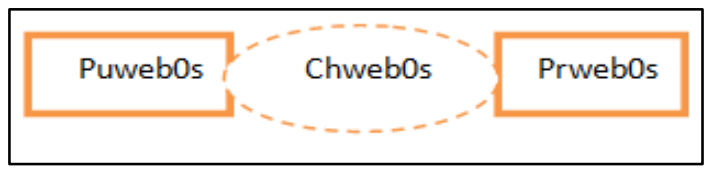

Figure 5. Blended of prwebOS and puWebOs create chwebOS

In order to putting into practice, the Web0S or Cloud operating system, it must be assess over/with a number of different kinds of computers (hardware and software), the (computer/server) $\mathrm{C} / \mathrm{S}$ should be more powerful than the client side in the respect of capacity and performance, because of the rule of networking and data communication support of the better in capacity and performance. The capability of the $\mathrm{C} / \mathrm{S}$ computer must be appropriate means that server must be powerful for solving the problem of traffic network (Bottleneck) network. Normally puWebOs is with LAMP and its more powerful than our PCs that connected to the server, also the server of the windows is higher capacity then our PCs in the net. The rate of traffic network (bottle neck) in both of them are roughly is same because the distance between client and server is a main factors. In general, Linux could be better because of it's an open source and free and easy to down load due to the compatibility in XAMP. Linux unlike windows because windows OS is return to a single company called (Microsoft), but Linux is not return to single company but varieties of companies produce Linux operating system under several names. Thus, the windows are from single company but linux is from multiple companies. Table (1) designates totally software and applications that used for designing tested system.

Table (1) describes all software and applications that used for designing proposed system

\begin{tabular}{|c|c|}
\hline Software interface1 & Software interface 2 \\
\hline Development server- XAMP & Development server- LAMP \\
\hline $\mathrm{X}$ - is cross platform & L- is Linux \\
\hline A- is apache & A- is windows \\
\hline M- is MySQL & M- is MySQL \\
\hline $\mathrm{P}$ - is PHP & $\mathrm{P}$ - is PHP \\
\hline Backend: database- MySQL & Backend: database- MySQL \\
\hline Frontend: HTML,CSS,JavaScript & Frontend: HTML,CSS,JavaScript \\
\hline Scripting Lanuage : PHP & Scripting Lanuage : PHP \\
\hline Applications: notepad ++ & Applications: notepad ++ \\
\hline
\end{tabular}

\subsection{Hardware Interfaces}

The hardware specifications of depended tools includes: (hosts, switches-hub and medium communication cables) in this system are shown in tables (2) and (3). In order to provide more practical applications and to be close to the real situation, the system has been implemented using hosts with different features and abilities. Table (2) shows shows totally hardware tools withspecification for the planned system mechanism. Also, Table (3) shows the specification of switch and cables depended in the planned system.

Table (2) shows totally hardware tools with specification for the

\begin{tabular}{|c|c|c|c|}
\hline \multicolumn{5}{c|}{ planned system mechanism } \\
\hline $\begin{array}{c}\text { PrwebOS } \\
\text { Server }\end{array}$ & Intel (R) $1.40 \mathrm{~GB}$ & $32 \mathrm{bits}$ & $2 \mathrm{~GB}$ \\
\hline Clients & Intel (R) $1.40 \mathrm{~GB}$ & 32 bits and 64 bits & $2 \mathrm{~GB}$ \\
\hline
\end{tabular}

Table (3) shows the specification of switch and cables depended in the

\begin{tabular}{|c|c|}
\hline \multicolumn{2}{|c|}{ planned system } \\
\hline Properties & Switch \\
\hline Name & Hup \\
\hline Number of ports & 32 \\
\hline Properties & Cable \\
\hline Type & Wireless \\
\hline Maximum & 100 MBYTE \\
\hline
\end{tabular}




\subsection{Time-loading Analysis Criteria:}

The developed criteria in this research are established web0S system by using of different methods namely; prWebOS Windows10 operating system and puWebOS-Linux operating system. These implementation results deal with two architectures of the proposed system. The results will be obtained using two types of OSs (Windows and Linux). Finally, results of this system will be evaluated by comparing them with a hand writing scripts in PHP(loading time). The evaluation placed in the computer-wireless using ten computers and smart devices as clients and a powerful computer as servers. The clients have different computer description (i.e. IPad, laptop, mobile) and connected via wireless local network. One time is prwebOS and the other time is puWebOs. The test is to find out which loading time is better when dealing with prwebOS.

Table (4) shows the total time and load time over different OS in seconds

\begin{tabular}{|c|c|c|c|}
\hline Web0S & Types & $\begin{array}{c}\text { Time } \\
\text { loading }\end{array}$ & $\begin{array}{c}\text { Total } \\
\text { time }\end{array}$ \\
\hline \multirow{3}{*}{ Lucid } & Non net- centric & $\mathbf{1 3 . 9 8}$ sec. & $\begin{array}{c}13.99 \\
\text { sec. }\end{array}$ \\
\cline { 2 - 4 } & $\begin{array}{c}\text { Windows / } \\
\text { prWebOS }\end{array}$ & $\begin{array}{c}\mathbf{2 6 . 9 5 6} \\
\text { sec. }\end{array}$ & $\begin{array}{c}26.957 \\
\text { sec. }\end{array}$ \\
\cline { 2 - 4 } & Linux / puWebOS & $\mathbf{3 . 8 4}$ sec. & 3.84 sec. \\
\hline
\end{tabular}

Generally, the total time (TT) and time loading(TL) is compared for each type of the network. The computer number that connected to the net-centric is a constant and its means that for all type of the investigation only utilize one computer as a client. The aims of it to show the total time and load time for (only one client) for TOS, PrWebOS and PuWebOS with different operating system Windows OS for TOS and PrWebOS, but Linux OS for PuWebOs. The least results TL and TT in seconds will be better for ordering from number one is Linux PuWebOS (3.84sec), number two is Non-centric $(13.98 \mathrm{sec})$ and number three is PrWebSs ( $26.995 \mathrm{sec}$ ). The ordering shows that the distance is not that important for web0S. The capacity of server and type of server is an important factor that realized. Accordingly, Linux OS is more suitable for WebOS. The PuWebOS is much powerful than the PrWebOS and TOS. The Figure (6) shows the loading time for lucid webOS.

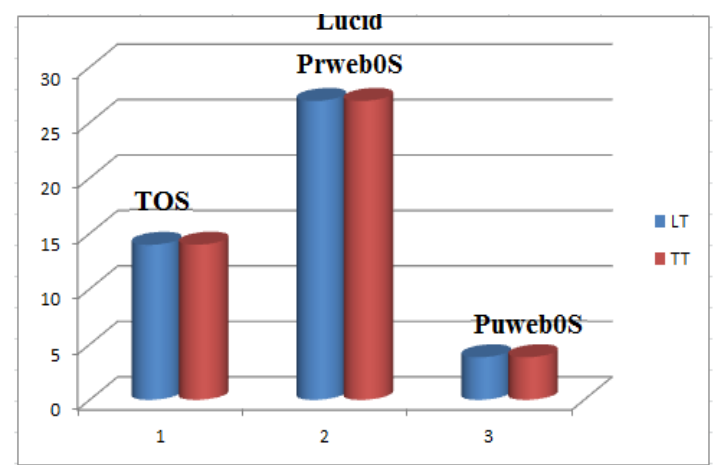

Figure 6. Shows loading time for lucid web0S

\section{RESULT ANALYSIS AND COMPARISON}

The overall evaluation of the proposed system shows that, the PuWebOs is perfectly reliable with optimum results for both mentioned webOS that is eyeOS and lucid. The index page of eyeOS is $(10 \mathrm{Kbyte}-3.44 \mathrm{sec})$ but the index page of lucid is $(13 \mathrm{Kbyte}-3.88 \mathrm{sec})$. The PrWebOS results is for both mentioned webOS that is eyeOS and lucid. The index page of eyeOS is $(10$ Kbyte $-15.90 \mathrm{sec})$, but the index page of lucid is (13 Kbyte-26.95). The TOS results is for both mentioned WebOS that is eyeOS and lucid. The index page of eye $0 \mathrm{~S}$ is (10Kbyte $-14.90 \mathrm{sec})$, but the index page of lucid is (13 Kbyte17.73). PuWebOS is better than PrWebOS and prWebOS is better than TOS. PuWebOS and PrWebOS are both suitable than TOS.

\section{CONCLUSION}

In this paper, the time loading benchmark is a very important due to find out the least time loading required over different operating systems namely Windows operating system (WOS) and Linux operating system (LOS). In one hand, the time loading over LOS-Ubuntu is required less time loading than WOS-Windows 10 . On the other hand, the security of LOSUbuntu is much higher than WOS-windows 10. Furthermore, LOS-Ubuntu is very easy to deal with if compared to WOSwindows 10 because the LOS is cross platform, open source and free. The previous section proved that PuWebOs is better than PrwebOS and prwebOS is better than TOS (N/A). PuWebOS and PrWebOS are both suitable then TOS because of that we mentioned that TOS is not applicable (N/A) for CC at all.

\section{REFERENCES}

A. Adekotujo,A. Odumabo,A. Adedokun,O. Aiyeniko, A Comparative Study of Operating Systems: Case of Windows, UNIX, Linux, Mac, Android and iOS, International Journal of Computer Applications (0975 - 8887) Volume 176 - No. 39, July 2020

A.Tripathi, "Web Operating Systems", Amity School of Engineering and Technology,Amity University, Noida, India 2014.

A.Vahdat, E.Belani, P.Eastham, C.Yoshikawa, T.Anderson, D.Culler, M.Dahlin,'WebOS: Operating System Services for Wide Area Applications", Computer Science Department, University of Texas, Austin, 1998.

Chunnjie LUO, Jianfeng ZHAN, Zhen JIA, Lei WANG, Gang LU, Lixin ZHANG, Chng-Zhong XU, Ninghui SUN, "Cloudrank-D: Benchmarking And Ranking Cloud Computing Systems For Data Processing Applications", Springer-Verlag Berlin Heidelberg 2012

Clouds with CloudWorkBench", International Conference on Performance Engineering Companion (ICPE '19 Companion), April 7-11, 2019, Mumbai, India, https://doi.org/10.1145/3302541.3310294.

D. Therrien, "Fact Sheet Cloud Computing", Office of the Privac Commissioner of Canada, May 2016.

D.Kirshna, R.Meshach, "Cloud Computing Along Web-OS”, Pollachi, International Journal of Computational Engineering Research,Vol, 03,Issue, 10, 2013.

H. Abusaimeh, "Cloud Web- Based Operating System (Cloud Web Os)", International Journal of Computer Science \& Information Technology (IJCSIT) Vol 6, No 2, April 2014.

H. Unger, P.Kroph," Overview About The Resource Scheduling In The Web Operating System(WOS ${ }^{\text {TM }, " ~ F B ~}$ Informatik,Universität Rostock, Canada, 2000.

H.Steen, Tiger, "Web Operating System for Modern Smartphones", Master of Science Thesis, Chalmers University Of Technology, Göteborg, Sweden, June 2011.

J.Duan, P.Fasker, A.Fesak, T.Stuart, "Benefits and Drawbacks of Cloud-Based ersus Traditioerp Systems", Tilburg University, Netherlands, 2012

Joel Scheuner, Philipp Leitner," Performance Benchmarking of Infrastructure-as-a-Service (IaaS)

L.Hasan, E. Abuelrub, "Assessing The Quality Of Web Sites", Department of Management Information Systems, Zarqa Private University, Jordan, 2011.

Mishra SK, Sahoo B, Parida PP Load balancing in cloud computing: a big picture. J King Saud Univ Comp Infor Sci:1-32, (2018) 
R. Sharma, "Applications of Web based Operating System" Department of Computer Science NITK, Surathkal, India, Volume: 1; Issue: 2; June - 2013

R.Mondal, D.Sarddar, "Web OS in CloudComputing: A Case study", Int. J. Advanced Networking and Applications, 2016

R.Sachdeva, P.Sharma, N.Kataria," Web Operating System Impediments", AP, Dept. of Computer Science, Dev Samaj College for Women, Ferozepur City, India 2015.

Rajat, Ajay Kumar Bharti, Performance Analysis of Cloud Computing, IJSRD - International Journal for Scientific
Research \& Development| Vol. 6, Issue 09, 2018 | ISSN (online): 2321-0613,2018.

Scot Schultz," Measuring modern high-performance computing", HPC/Technical Computing, Nvidia networking business unit, USA, October 19, 2020

Shubham kumar sahu, 2Dr. R.K. Khare,Survay on Web Based Operating System, JETIR, Volume 6, Issue 6, June 2019. 